\title{
Post-acquisition effects of viruses on vector behavior are important components of manipulation strategies
}

\author{
Quentin Chesnais ${ }^{1,2}$ (1) $\cdot$ Gabriela Caballero Vidal ${ }^{1} \cdot$ Roxane Coquelle $^{1} \cdot$ Michel Yvon $^{3} \cdot$ Kerry Mauck $^{4} \cdot$ \\ Véronique Brault ${ }^{2} \cdot$ Arnaud Ameline $^{1}$
}

Received: 9 July 2020 / Accepted: 19 September 2020

(c) Springer-Verlag GmbH Germany, part of Springer Nature 2020

\begin{abstract}
A growing number of studies suggest that plant viruses manipulate host plant phenotypes to increase transmission-conducive behaviors by vectors. Studies on this phenomenon frequently omit examination of interactions that occur after vectors acquire virions, which provides an incomplete understanding of the ecology of plant virus manipulation. Here, by taking a full factorial approach that considered both the infection status of the host (Montia perfoliata) and viruliferous status of the aphid (Myzus persicae), we explored the effects of a circulative, non-propagative virus (Turnip yellows virus [TuYV]) on a suite of behavior and performance metrics that are relevant for virus transmission. Our results demonstrate that viruliferous aphids exhibited an increased velocity of movement and increased activity levels in locomotor and dispersal-retention assays. They also had increased fecundity and showed a capacity to more efficiently exploit resources by taking less time to reach the phloem and ingesting more sap, regardless of plant infection status. In contrast, non-viruliferous aphids only exhibited enhanced fecundity and biomass on TuYV-infected hosts, and had overall reduced dispersal and locomotor activity relative to viruliferous aphids. In this pathosystem, post-acquisition effects were stronger and more conducive to virus transmission than the purely pre-acquisition effects mediated by virus effects on the host plant. Our study provides additional support for the hypothesis that virus manipulation of vector behavior includes both pre- and post-acquisition effects and demonstrates the importance of considering both components when studying putative virus manipulation strategies.
\end{abstract}

Keywords Aphid peformance $\cdot$ Behavioral phenotype $\cdot$ Pathogen transmission $\cdot$ Plant virus $\cdot$ Vector manipulation

Communicated by Jennifer Thaler.

Electronic supplementary material The online version of this article (https://doi.org/10.1007/s00442-020-04763-0) contains supplementary material, which is available to authorized users.

Quentin Chesnais

quentin.chesnais@inrae.fr

$\triangle$ Arnaud Ameline

arnaud.ameline@u-picardie.fr

1 UMR CNRS 7058 EDYSAN (Écologie et Dynamique des Systèmes Anthropisés), Université de Picardie Jules Verne, 33 Rue St Leu, 80039 Amiens Cedex, France

2 Institut National de Recherche en Agriculture, Alimentation et Environnement, SVQV UMR-A1131, Université de Strasbourg, 68000 Colmar, France

3 Institut National de Recherche en Agriculture, Alimentation et Environnement, BGPI, Université de Montpellier, SupAgro, CIRAD, 34000 Montpellier, France

4 Department of Entomology, University of California, Riverside, CA 92521, USA

\section{Introduction}

Plant viruses fundamentally change the physiology of their hosts. Virus-induced changes in host morphology, chlorophyll content, metabolism, defense, and many other phenotypic aspects can have drastic effects on plant productivity and fruit quality (Culver and Padmanabhan 2007). Although these negative outcomes have driven much of the research on virus symptom expression, accumulating evidence suggests that symptoms induced by vector-borne viruses may also be important components of virus ecology and epidemiology. Foraging and feeding behaviors of mobile insect vectors responsible for virus transmission are strongly influenced by the same phenotypic aspects altered during virus infection (Fereres and Moreno 2009; Mauck et al. 2018; Eigenbrode et al. 2018). Since vectors are essential for initiating new infections, it is logical to hypothesize that selection will favor viruses inducing phenotypes that enhance the 
probability of vectors visiting infected hosts and performing behaviors conducive to virus acquisition and retention.

This 'manipulation' of the host by the virus to encourage interactions with vectors is not a concept that is unique to plant viruses. Similar phenomena are well documented among eukaryotic parasites that rely on trophic interactions among different species for transmission and life cycle completion (Lefèvre et al. 2009; Poulin et al. 2010). Parasitic acanthocephalans, cestodes, nematodes, and trematodes can modify the activity and microhabitat preferences of their intermediate hosts (often an invertebrate) in ways that make them more likely to be predated upon by the final host in the parasite life cycle (often a vertebrate) (Lafferty and Shaw 2013). Despite plant viruses being much simpler organisms relative to multicellular parasites, a growing number of studies suggest that symptoms of virus infection may not be mere by-products of pathology, but instead, the result of adaptations for encouraging vector contacts with infected hosts and virion acquisition (Mauck et al. 2012, 2016). For example, increased attraction of vectors to infected hosts has been documented in response to changes in odor-based and visual plant phenotypes elicited by plant viruses in at least four families (Bromoviridae, Potyviridae, Luteoviridae, and Reoviridae) (Eigenbrode et al. 2002; Jimenez-Martinez et al. 2004; Mauck et al. 2014; Lu et al. 2016; Claudel et al. 2018).

An even greater number of studies across a wider diversity of taxa report that plant virus infections enhance the specific behaviors required for vectors to acquire and retain virions after contacting infected hosts (reviewed in Mauck et al. 2012, 2018). Increases in transmission-conducive probing and feeding behaviors occur when viruses induce changes in phenotypic aspects influencing plant palatability (e.g., leaf physical characteristics, primary metabolites, secondary metabolites, and inducible defenses) (McMenemy et al. 2012; Casteel et al. 2014; Chesnais et al. 2019a). In contrast to virus effects on host attractiveness to vectors, modifications to host palatability components are not uniform, but seem to vary in a predictable way depending on the transmission mechanism requirements of each virus taxon; viruses acquired and inoculated through brief probes of epidermal tissue followed by rapid dispersal tend to reduce palatability, while viruses acquired and inoculated through long-term feeding (hours to days) in plant phloem elements tend to increase palatability, ease of phloem access, and ease of phloem sap ingestion (Mauck et al. 2012, 2018). This dichotomy of virus effects, which is driven by shared transmission characteristics and not virus phylogeny, lends support to the idea that plant virus effects on host phenotypes and vector behavior may be adaptive. This supposition is further supported by recent functional genomics studies identifying virus proteins and conserved host pathways involved in manipulations (Mauck et al. 2019; Ziegler-Graff 2020).
Documentation of virus effects on host phenotypes is important for establishing that such effects may constitute adaptive manipulations by viruses, but it largely ignores the important interactions that occur after vectors contact infected hosts and acquire virions. For example, most of the viruses purported to manipulate host plant phenotypes are circulative viruses, meaning virions traverse the vector gut barrier to form long-term, persistent associations with vector tissues (e.g., salivary glands), where they can reside (circulative, persistent, non-propagative), and even replicate (circulative, persistent, propagative) (Hogenhout et al. 2008). Thus, acquisition of virions by a vector insect presents yet another target organism for phenotypic manipulation. The resulting "direct effects" of viruses on vector behavior and physiology, which occur after acquisition, can be considered mechanistically distinct from the "indirect effects" described above because they do not involve a plant host as the intermediary between the virus and vector (Mauck et al. 2019).

Direct manipulation of vector behavior in ways that enhance transmission has now been documented in the laboratory for several circulative plant viruses, and the epidemiological consequences of these observations have been modeled at field scales (Roosien et al. 2013; Shaw et al. 2017). The handful of empirical studies to date indicate that virus acquisition and retention often significantly influence subsequent vector orientation and settling preferences in ways that should increase virus transmission (reviewed in Mauck et al. 2018, 2019). For example, Myzus persicae (Sulzer) aphids that had acquired potato leafroll virus (PLRV, Luteoviridae) from infected plants preferred to orient towards volatiles from non-infected potatoes, while non-viruliferous aphids preferred volatiles from PLRV-infected plants (Rajabaskar et al. 2014). The same preference reversal was evident when aphids had access to contact-based cues; viruliferous aphids settled preferentially on healthy plants, while non-viruliferous aphids settled more often on infected plants. Theoretical extrapolations of these effects to field scales suggest that reversals in vector preferences following virus acquisition are beneficial for virus spread, and, in some cases, may be essential components of the vector manipulation process (Roosien et al. 2013; Shaw et al. 2017). There is even evidence that direct effects of viruses go beyond movement and dispersal to exert subtle changes in feeding behaviors that are closely linked with virus inoculation (e.g., salivation and ingestion), which can be measured for many vector insects using the Electrical Penetration Graph (EPG) technique (Tjallingii 1988). In two of the earliest direct manipulation studies, Stafford et al. (2011) reported an increase in salivation events responsible for virus inoculation for thrips infected with the circulative, propagative tomato spotted wilt virus (Tospoviridae) (TSWV). And, Moreno-Delafuente et al. (2013) reported increased durations of phloem contacts and salivation among whiteflies carrying the circulative, 
non-propagative tomato yellow leaf curl virus (Geminiviridae) (TYLCV), as well as more rapid movement of viruliferous vectors. Both alterations are expected to facilitate the inoculation and establishment of respective viruses in susceptible host plants; TSWV through extended salivation in epidermal and mesophyll cells (Rotenberg et al. 2009; Stafford et al. 2011), and TYLCV through extended salivation into phloem sieve elements (Prado and Tjallingii 1994).

The studies discussed above support the hypothesis that virus manipulation of vectors includes direct, post-acquisition effects on behavior. But most focus on one or two aspects of post-acquisition vector biology without considering additional dimensions that strongly influence the probability of a viruliferous vector successfully dispersing to, and inoculating, a susceptible host plant. This provides an incomplete understanding of the ecology of manipulation for key pathosystems and leaves out details that are necessary to parse the mechanisms by which viruses are able to directly manipulate vectors. To address this, we explored the effects of a circulative, non-propagative virus (Turnip yellows virus [TuYV], genus Polerovirus family Luteoviridae) on a suite of vector traits that are relevant to transmission, including host preferences, in-leaf feeding behavior, locomotor activity, fecundity, and biomass. We previously documented that TuYV induces changes in host plant phenotype consistent with indirect vector manipulation (Chesnais et al. 2019a, b). Here, we provide evidence that TuYV manipulation extends beyond the period of time where the vector is in contact with the infected host, while demonstrating the importance of considering multiple axes of vector behavior and physiology in direct effects studies.

\section{Materials and methods}

\section{Study system}

Seeds of claytonia (Montia perfoliata cv. "Claytone de Cuba", Caryophyllales: Portulaceae) provided by 'Graines Baumaux' (Mazirot, France) were sown in pots $(60 \times 60 \times 65 \mathrm{~mm})$ containing commercial sterilized potting soil and maintained in a growth chamber under $20 \pm 1{ }^{\circ} \mathrm{C}$, $60 \% \pm 5 \%$ relative humidity (RH), and $16 \mathrm{~L}: 8 \mathrm{D}$ photoperiod at 2.5 klux.

The Myzus persicae (Sulzer) (Hemiptera: Aphididae) colony was established from one parthenogenetic female collected in 1999 in a potato field near Loos-en-Gohelle (France). The colony was reared on rapeseed (Brassica napus cv. "Adriana") (Brassicales: Brassicaceae). Pots $(90 \times 90 \times 100 \mathrm{~mm})$ containing each 3-4 rapeseed plants were placed in ventilated plastic cages $(240 \times 110 \times 360 \mathrm{~mm})$ and maintained in a growth chamber under the conditions described above. For all the experiments, apterous adult
( $8 \pm 1$ day old) aphid clones were synchronized on rapeseed leaves set in 1.5\% agar in Petri dishes (90 mm diameter). Prior to the experiments, these synchronized adult aphids were placed for $24 \mathrm{~h}$ on TuYV-infected or non-infected $M$. perfoliata, to create two groups of aphids: the viruliferous aphids (i.e., aphids carrying the virus) and the nonviruliferous aphids. Although 'viruliferous' aphids were not tested to confirm the presence of the virus, after 24-h feeding on TuYV-infected plants, in our many years of experience with this system, we have found that the likelihood of TuYV transmission to a susceptible host by one Myzus persicae following a $24-\mathrm{h}$ acquisition access period is close to $100 \%$ (unpublished data).

The turnip yellows virus (TuYV) used in our experiments was provided by Véronique Ziegler-Graff at IBMPCNRS (Strasbourg, France) and maintained on $M$. perfoliata (Leiser et al. 1992). Plants were inoculated with TuYV by placing two aphids, previously allowed a 24-h acquisition access period on TuYV-infected $M$. perfoliata, on a single 14-day-old plant for a 3-day inoculation period. After $72 \mathrm{~h}$, aphids and nymphs were gently removed with a paintbrush. The infection status of the inoculated plants was visually confirmed 21 days post-inoculation (dpi) by symptoms observation: dwarfing, reddening/yellowing of leaf margins and interveinal discoloration. Virus infection was also confirmed using double antibody sandwich enzyme-linked immunosorbent assay with polyclonal antibodies produced by LOEWE (Adams and Clark 1977). Sham-inoculated (i.e., non-infected) plants were treated similarly using non-viruliferous aphids. For all the bioassays described below, plants were used three weeks after virus inoculation or sham inoculation. All experiments were conducted under controlled conditions $\left(20 \pm 1{ }^{\circ} \mathrm{C}, 60 \% \pm 5 \%\right.$ relative humidity $[\mathrm{RH}]$, and 16 L: 8 D photoperiod at 2.5 klux).

\section{Aphid locomotor activity}

Dispersion behavior and velocity of viruliferous and nonviruliferous aphids were monitored on a target arena (Fig. 1). One by one and alternately, an aphid, either viruliferous or not, was deposited in the center of a paper arena $(285 \mathrm{~mm}$ diameter), divided into 10 concentric circles ("spatial zone") spaced by $15 \mathrm{~mm}$. Aphids were deposited on a transparent glass plate placed on the paper arena, which allowed cleaning of the glass surface with ethanol every 5 aphids. To minimize external stimuli, the arena was positioned between 4 white foam cardboard "walls" and a white fluorescent tube was positioned above. For each aphid, we determined the number of "spatial zones" crossed (parameter "movement"), the maximum zone reached (parameter "dispersion"), and the time taken to move from one zone to another for a maximum of $300 \mathrm{~s}$ (parameter "velocity"). The test is completed if (i) the aphid crosses the 10th spatial zone and 


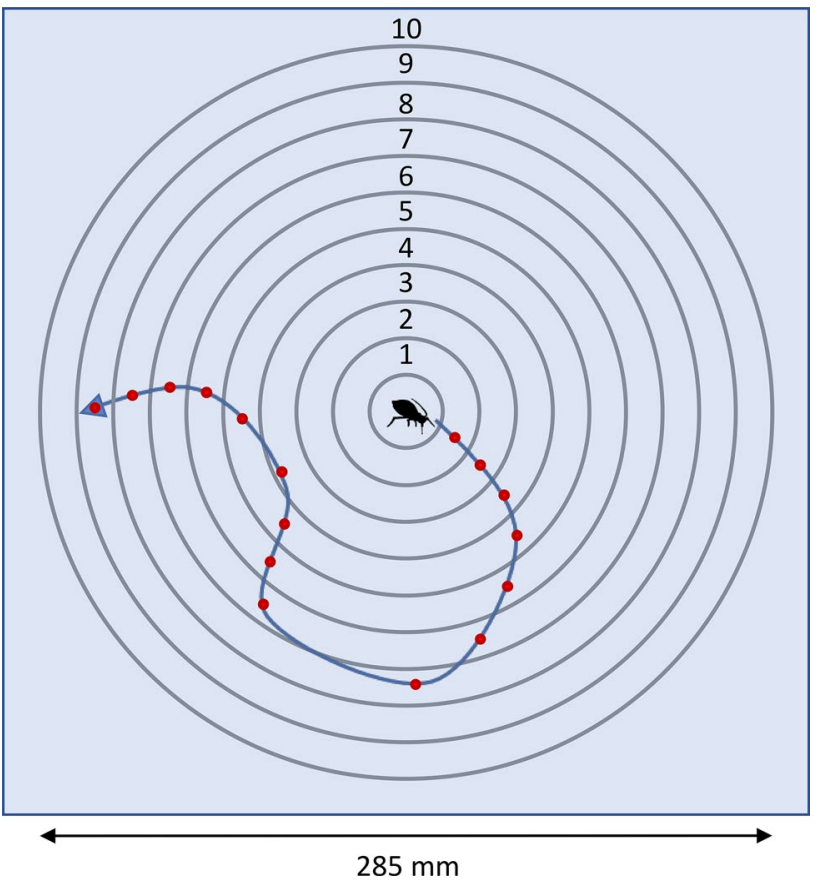

Fig. 1 Bioassay set-up used to record dispersion behavior and locomotor activity of aphid (paper arena covered with a squared glass plate). Blue line: aphid path on the arena in $300 \mathrm{~s}$; red dots: number of spatial zones in which the aphid has entered ("movement"). The maximum spatial zone reached is considered a "dispersion" parameter. The "velocity" parameter is equal to the average duration in each zone

leaves the arena, or (ii) at the end of $300 \mathrm{~s}$. Two blocks of twenty aphids were used for each aphid status (viruliferous or non-viruliferous).

\section{Aphid settlement preference and migration}

Aphid emigration and settlement preference were tested in arenas allowing contact-based, odor-based, and visual cues. The experimental set-up used was adapted from Mauck et al. (2010) (Fig. 2). In these bioassays, we assessed the propensity of apterous aphids (either viruliferous or not) to emigrate from infected or sham-inoculated plants. Ten aphids were released onto leaves of an infected or sham-inoculated plant (the "release" plant) placed adjacent to a second plant (the "choice" plant), which was of the opposite status. The two plants in the cage were linked by a bridge allowing aphids to move between plants. The whole setting was placed in a $360 \times 240 \times 110 \mathrm{~mm}$ plastic and aerated cage where the "release" and "choice" plants were randomly placed to avoid any position effect. To minimize external stimuli, the cage was positioned between 4 white foam cardboard walls and a white fluorescent tube was positioned above. Aphids were then counted on each plant $24 \mathrm{~h}$ after deposition. Each test was repeated 15 times.

\section{Aphid feeding behavior}

We used the electrical penetration graph DC system as described by Tjallingii (1988) to investigate the effects of both (i) the plant status (TuYV infected or sham inoculated) and (ii) the aphid status (viruliferous or not) on the feeding behavior of $M$. persicae. We created electrical circuits that each included one aphid and one plant by tethering a thin gold wire $(20 \mu \mathrm{m}$ diameter and $2 \mathrm{~cm}$ long) on the insect's dorsum using conductive silver glue (EPG systems, Wageningen, the Netherlands). To facilitate the tethering process, aphids were immobilized at the edge of a pipette tip using a vacuum pump (KNF Neuberger, model. N86KN.18. Germany). Eight apterous aphids were connected to the Giga-8 DC-EPG amplifier and each one was placed on the leaf (adaxial side) of an individual plant. A second electrode was inserted into the soil of each potted plant to close the electrical circuit. The recordings were performed continuously for $8 \mathrm{~h}$ during the photophase. Each aphid-plant system was placed inside a Faraday cage at $20 \pm 1{ }^{\circ} \mathrm{C}$. Acquisition and analysis of the EPG waveforms were carried out with PROBE 3.5 software (EPG-Systems, www.epgsy stems.eu). Relevant aphid behavior EPG parameters were calculated with EPG-Calc 6.1 software (Giordanengo 2014) and were based on different EPG waveforms described by (Tjallingii and Hogen Esch 1993). We decided to select a few relevant and important parameters for the acquisition of TuYV by aphids, i.e., the total durations of "probing time" and "phloem sap ingestion phases" and the time needed by the aphid to reach the phloem (additional parameters regarding pathway phases and phloem salivation are included in Table S1). In this study, the feeding behavior of viruliferous and non-viruliferous $M$. persicae on $M$. perfoliata, infected or not by TuYV, was investigated using 19-23 individuals for each "aphid" $x$ "plant" combination (Non-viruliferous feeding on sham-inoculated plant, $n=22$; Non-viruliferous feeding on TuYV-infected plant, $n=20$; Viruliferous feeding on sham-inoculated plant, $n=23$; Viruliferous feeding on TuYV-infected plant, $n=19$ ).

\section{Aphid fecundity and biomass}

Synchronized apterous adults ( $8 \pm 1$ day old) were randomly selected from the pools of viruliferous and non-viruliferous aphids and transferred onto plants of TuYV-infected or sham-inoculated $M$. perfoliata plants to study adult fecundity. Each adult aphid was individually placed in a clip cage. The number of nymphs produced was recorded daily for 4 days. New nymphs were removed and counted daily with a soft camel-hair brush to estimate the daily fecundity of each individual parent. For each combination of plant status (TuYV infected or sham inoculated) per aphid status (viruliferous or non-viruliferous), 19-20 aphids were used. 
(a)
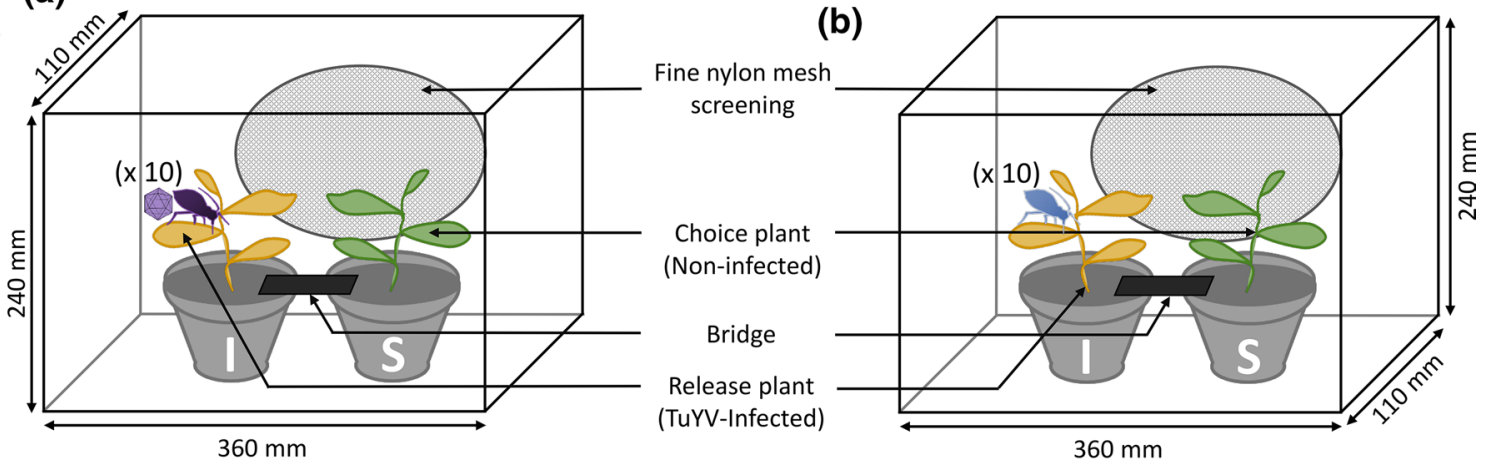

(c)
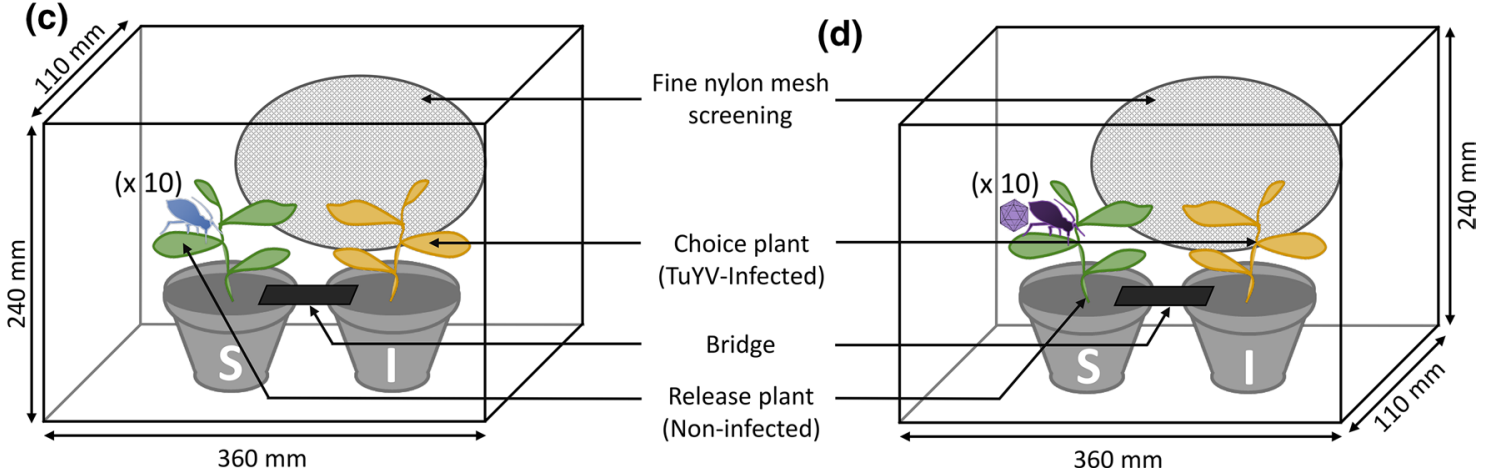

Fig. 2 Bioassay set-up used to test aphid emigration and settlement on infected and non-infected plants. a Viruliferous Myzus persicae (purple) released on infected Montia perfoliata (yellow); b Non-viruliferous M. persicae (blue) released on infected M. perfoliata; $\mathbf{c}$ Non-

Groups of synchronized first-instar nymphs (less than $24 \mathrm{~h}$ old) of $M$. persicae were obtained from parthenogenetic adult females placed on either TuYV-infected or non-infected M. perfoliata leaves set in 1.5\% agar in Petri dishes ( $90 \mathrm{~mm}$ diameter). After $24 \mathrm{~h}$, the adult females were removed from the petri dishes (three per modality) and the newly deposited $M$. persicae remained. To quantify effects of TuYV infection on aphid biomass, ten 4-day-old juvenile aphids per Petri dish (thirty aphids total per modality) were randomly selected, frozen at $-80^{\circ} \mathrm{C}$ and subsequently weighted individually, using a precision electronic scale (Mettler M3, class 1, Max: $3 \mathrm{~g}$, Low: $1 \mu \mathrm{g}, T=-3 \mathrm{G}$ $[\mathrm{dd}]=1 \mu \mathrm{g}$ ).

\section{Statistical analyses}

Locomotor activity parameters and aphid fecundity were analyzed with a non-parametric ANOVA using ranks (package R: "Rfit"; $p$ value adjustment with "fdr" method) because homogeneity of variances (Levene test) and normality (Shapiro-Wilk test) were not verified. When a significant effect of one of the main factors was detected or when an interaction between factors was significant, non-parametric multiple comparisons for relative effects (package R: viruliferous $M$. persicae released on sham-inoculated $M$. perfoliata (green) and $\mathbf{d}$ Viruliferous $M$. persicae released on sham-inoculated M. perfoliata. (S: sham-inoculated plant; I: infected plant)

"nparcomp") ( $p$ value adjustment with "Tukey" method) at the 0.05 significance level was used to test for differences between treatments. Data on aphid retention/dispersion were analyzed with a non-parametric ANOVA using permutations on values (package R: "permuco"; freedman_lane to handle nuisance variables and 50.000 permutations; $p$ value adjustment with "fdr" method) because normality (Shapiro-Wilk test) was not verified.

We used generalized linear models (GLM) with a likelihood ratio and Chi-square test to assess whether there was an effect of plant or aphid status on $M$. persicae feeding behavior. When a significant effect of one of the main factors was detected or when an interaction between factors was significant, a pairwise comparison using least-squares means (package R: "lsmeans") ( $p$ value adjustment with Tukey method) at the 0.05 significance level was used to test for differences between treatments. Data on aphid feeding behavior (probing and phloem sap ingestion phases) was not normally distributed, so we carried out a GLM using a Gamma (link = "inverse") distribution. When a significant effect of one of the main factors was detected or when an interaction between factors was significant, a pairwise comparison using least-squares means (package R: "lsmeans") ( $p$ value adjustment with Tukey method) at the 0.05 
significance level was used to test for differences between treatments. Data on aphid feeding behavior $(t 1<\mathrm{E} 2)$ were modeled using the Cox proportional hazards $(\mathrm{CPH})$ model and we treated cases where the given event did not occur as censored. The assumption of validity of proportional hazards was checked using the functions "coxph" and "cox. zph", respectively (package R: "survival"). When a significant effect of one of the main factors was detected or when an interaction between factors was significant, a pairwise comparison using Estimated Marginal means (package R: "emmeans") (p-value adjustment with Tukey method) at the 0.05 significance level was used to test for differences between treatments. The fit of all generalized linear models was controlled by inspecting residuals and QQ plots. Data on aphid biomass were analyzed using a Wilcoxon test because normality was not verified (Shapiro test). All statistical analyses were performed using the $\mathrm{R}$ software (version 3.3.2) ( $\mathrm{R}$ Core Team 2019).

\section{Results}

\section{Aphid locomotor activity}

The velocity of viruliferous aphids was increased compared to non-viruliferous aphids (i.e. shorter duration spent per spatial zone in the arena) (ANOVA on aphid status, $F=51.35$, $P<0.001$ ) for both experiment blocks (Fig. 3). We also observed overall locomotor activity varied significantly across experiment blocks, (ANOVA, $F=16.72, P<0.001$ ) and a significant interaction between experiment block and aphid status (ANOVA, $F=20.10, P<0.001$ ). However, the overall effect of viruliferous status was maintained cross blocks: non-viruliferous aphids took on average between 40 and $70 \mathrm{~s}$ to cross

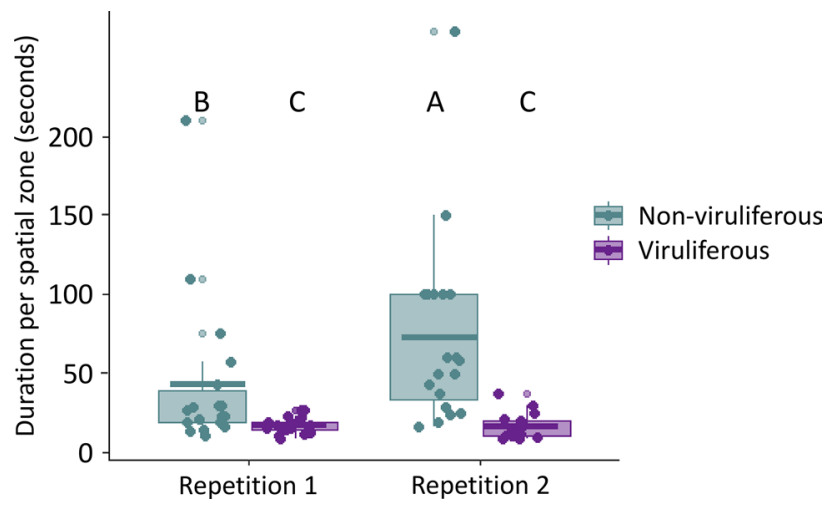

Fig. 3 Locomotor activity (duration per spatial zone in seconds) of TuYV-viruliferous and non-viruliferous $M$. persicae. Box plots show median (line), 25-75\% percentiles (box), 10-90\% percentiles (whisker), and outliers (dots). Letters indicate significant differences between aphid status associated with non-parametric ANOVA followed by non-parametric multiple comparisons a spatial zone, while the viruliferous aphids were 3-4 times faster $(\sim 16-17 \mathrm{~s})$.

\section{Aphid settlement preference and migration}

Viruliferous aphids were less likely to remain on the "release" plants and dispersed more often to the "choice" plants relative to non-viruliferous aphids (ANOVA; retention: $P<0.001$; dispersion: $P<0.001)$. This increased dispersal was present regardless of the plant status (ANOVA; retention: $P=0.050$; dispersion: $P=0.074$ ) (Fig. 4). We did not detect an interaction between aphid viruliferous status and plant infection status (ANOVA; retention: $P=0.509$; dispersion: $P=0.912$ ).

\section{Aphid feeding behavior}

The duration of general probing phase in plant tissue was not affected by the aphid status (GLM, $d f=81, F=3.271$, $P=0.074)$ nor the plant status (GLM, $d f=82, F=0.184$, $P=0.669$ ) (Fig. 5a). Time to first phloem phase was significantly influenced by aphid status (Cox model, $\chi^{2}=13.877$, $P<0.001$ ) (Fig. 5b), plant status (Cox model, $\chi^{2}=7.752$, $P=0.005$ ) and their interaction (Cox model, $\chi^{2}=5.028$, $P=0.025)$. Overall, Time to first phloem phase was significantly higher when non-viruliferous aphids were on TuYV-infected M. perfoliata plants (Fig. 5b). The duration of phloem sap ingestion phases was longer for viruliferous aphids than non-viruliferous aphids (GLM, $d f=63$, $F=6.402, P=0.014$ ) (Fig. 5c), regardless of plant status (GLM, $d f=64, F=1.220, P=0.273$ ). There were no significant interactions between aphid status and plant status terms for both probing and phloem sap ingestion duration parameters (GLM, $P>0.05$ ).

\section{Aphid fecundity and biomass}

Aphid fecundity was significantly influenced by aphid status (ANOVA, $F=40.35, P<0.001$ ), plant status (ANOVA, $F=12.85, P<0.001$ ) and their interaction (ANOVA, $F=12.14, P<0.001$ ). Overall, aphid fecundity was significantly higher when aphids were either viruliferous (regardless of plant status) or reared on TuYV-infected M. perfoliata plants (Fig. 6a). When reared on infected plants, the biomass of aphids increased by $50 \%$ compared to aphids reared on sham-inoculated $M$. perfoliata plants (Wilcoxon rank sum test, $W=108.5 ; P<0.001$ ) (Fig. 6b).

\section{Discussion}

Aphid feeding and movement are influenced by visual, odor-based, physical, and gustatory host cues conveying information about suitability (Margaritopoulos et al. 2005; 
Fig. 4 Aphid behavioral responses to contact, volatile and visual cues of sham-inoculated (i.e., non-infected) and TuYV-infected $M$. perfoliata plants after $24 \mathrm{~h}$. Ten viruliferous or non-viruliferous aphids were allowed to disperse from leaves of a non-infected or infected "release plant" to an adjacent "choice plant" of the opposite viral infectious status (mean \pm SEM). Fifteen replicates were performed for each condition. The asterisks indicate a significant difference between viruliferous and non-viruliferous $M$. persicae (ANOVA, $* * * P<0.001)$
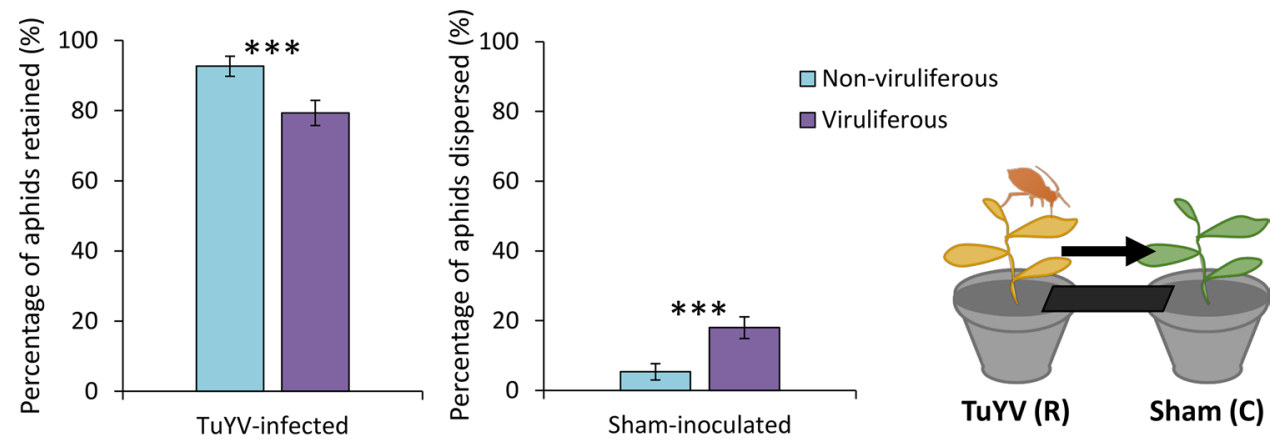

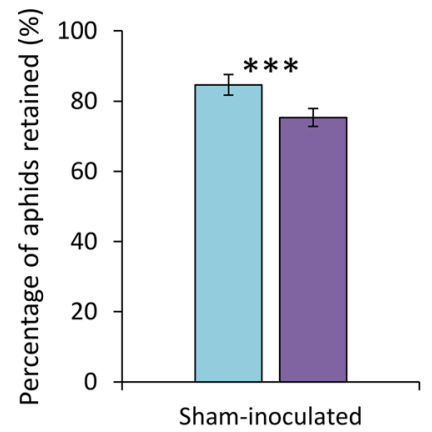

Release plant (R)

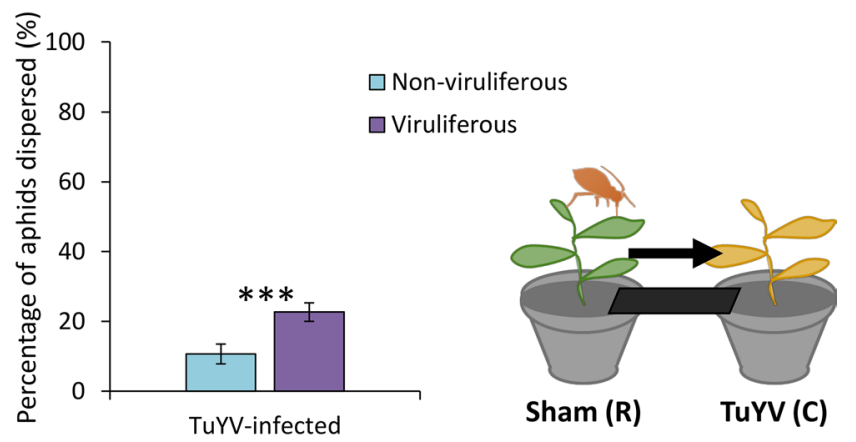

Choice plant (C) (a)

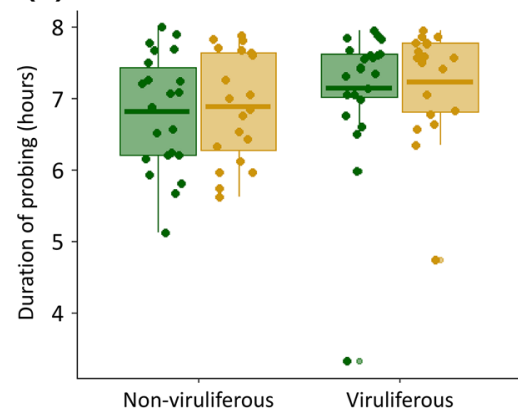

(b)

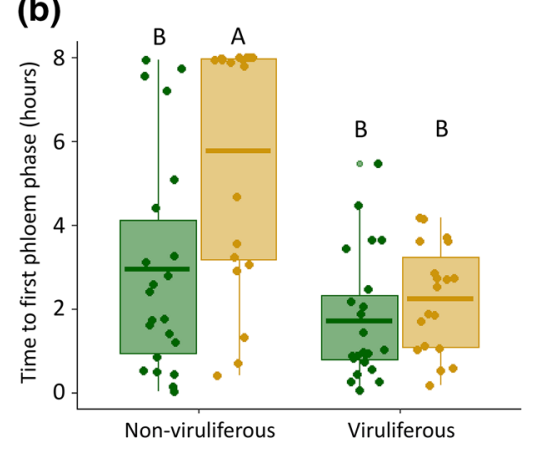

(c)

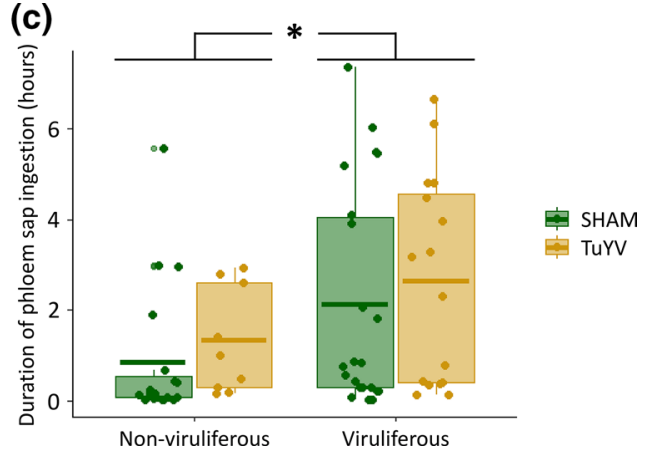

Fig. 5 Feeding behavior variables of viruliferous and non-viruliferous $M$. persicae on $M$. perfoliata plants (TuYV infected or sham inoculated). a Duration of probing phase in plant tissue; b Time to first phloem phase and c Duration of phloem sap ingestion. Box plots show median (line), 25-75\% percentiles (box), 10-90\% percentiles (whisker), and outliers (dots). Letters show significant dif-

Powell et al. 2006). Plant virus effects on host plant cues frequently elicit changes in the durations and frequencies of vector feeding or movement behaviors in ways that are likely to enhance transmission (Fereres and Moreno 2009; Mauck et al. 2012). Consistent with this, our results demonstrate that $M$. perfoliata host plant phenotype is altered by TuYV infection in ways that are likely to enhance transmission by non-viruliferous vectors contacting infected plants for the first time. However, by carrying out concurrent experiments with viruliferous insects, we provide evidence that the transmission-conducive effects of TuYV on aphid vector behavior extend far beyond the acquisition ferences between aphid viruliferous status and plant infection status as tested with Cox model followed by pairwise comparisons using 'emmeans' (method: Tukey). The asterisks indicate a significant difference between viruliferous and non-viruliferous $M$. persicae (GLM, $* P<0.05)$

access period. Aphids that fed on TuYV-infected M. perfoliata for $24 \mathrm{~h}$ prior to being used in experiments showed increased velocity of movement (time to cross spatial zones in an arena) and increased activity levels (movement among hosts) in locomotor and dispersal-retention assays (Fig. 7). Viruliferous aphids also had increased fecundity and showed a capacity to more efficiently exploit resources by taking less time to reach the phloem and ingesting more sap (Fig. 7). Surprisingly, fecundity and feeding efficiency effects occurred regardless of whether the host plant was infected with TuYV or sham inoculated (Fig. 7). In contrast, non-viruliferous aphids only exhibited enhanced 
(a)

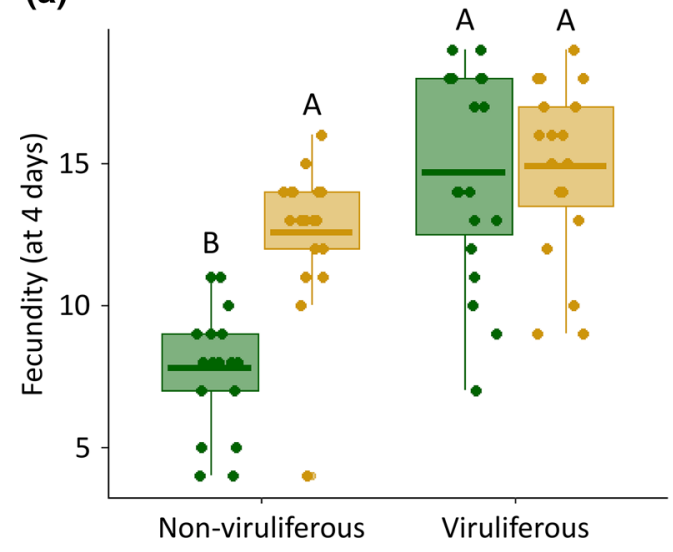

Fig. 6 Performance parameters of $M$. persicae reared on sham-inoculated or TuYV-infected $M$. perfoliata plants. a Fecundity (at 4 days) of viruliferous or non-viruliferous $M$. persicae. b Biomass of fourday-old $M$. persicae aphids. Box plots show median (line), 25-75\% percentiles (box), 10-90\% percentiles (whisker), and outliers (dots).

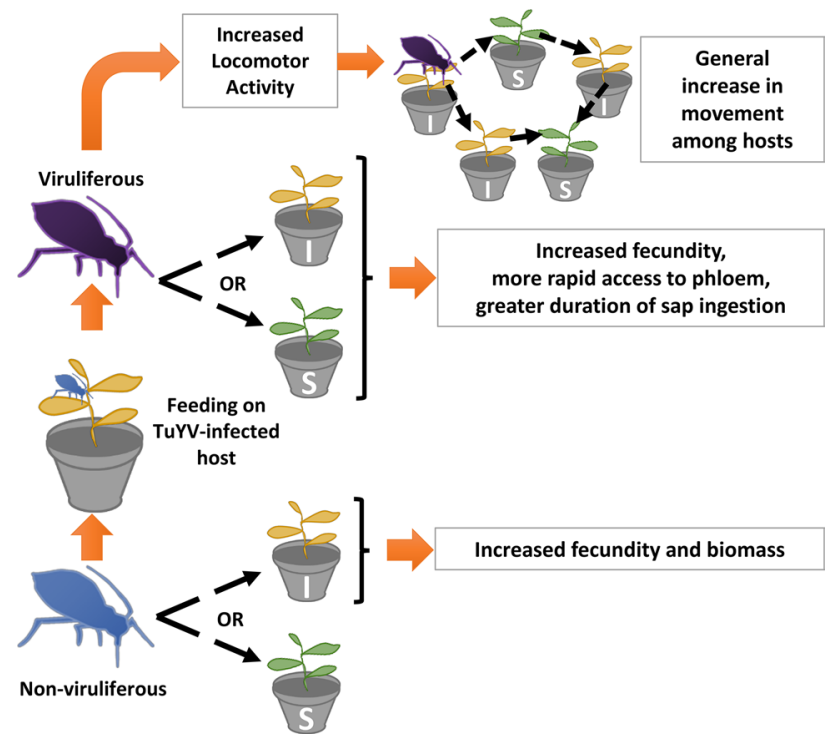

Fig. 7 Conceptual figure showing the observations for TuYV pre- and post- acquisition effects on vector behavior and performance. Blue aphid=non-viruliferous Myzus persicae; purple aphid=viruliferous $M$. persicae; green plant $=$ sham-inoculated Montia perfoliata $(\mathrm{S})$; yellow plant $=\mathrm{TuYV}$-infected $M$. perfoliata $(\mathrm{I})$

fecundity and biomass on TuYV-infected hosts, and had overall reduced dispersal and locomotor activity relative to viruliferous aphids (Fig. 7). Our study provides additional support for the hypothesis that virus manipulation of vector behavior includes both pre-acquisition effects mediated by the host and post-acquisition effects, which may be a product of viruses directly altering physiological aspects of the vector that are important for behavior (Mauck et al. 2019). (b)

SHAM TuYV

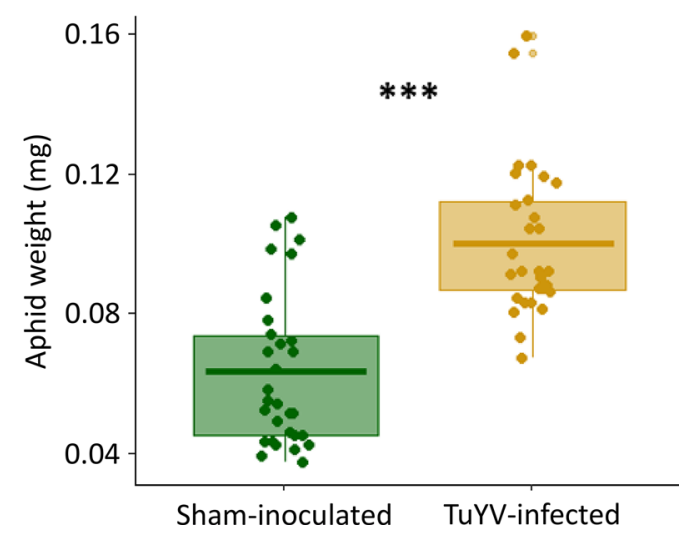

Letters indicate significant differences between aphid status associated with non-parametric ANOVA followed by non-parametric multiple comparisons. The asterisks indicate a significant difference between TuYV-infected and sham-inoculated $M$. perfoliata plants (Wilcox rank sum test, $* * * P<0.001$ )

Although post-acquisition effects of persistent, circulative viruses on vector behavior have been noted previously, several of these studies did not measure responses of viruliferous vectors to both infected and non-infected hosts. Notably, studies employing the Electrical Penetration Graph (EPG) technique report that viruliferous whiteflies and thrips engage in transmission-conducive behaviors on non-infected hosts (Stafford et al. 2011), as well as hosts that are not susceptible to the virus (Moreno-Delafuente et al. 2013), but not on infected hosts. However, using a full factorial design in our EPG study, we showed a significant interaction between aphid viruliferous status and plant infection status for the time it takes for insects to reach the phloem, a critical metric for both virus transmission and aphid nutrition (Fig. 5) (Prado and Tjallingii 1994; Fereres and Moreno 2009). Non-viruliferous aphids take significantly longer to reach the phloem on TuYV-infected M. perfoliata plants (an effect which is somewhat detrimental for both aphid and virus), but viruliferous aphids do not encounter this same difficulty, and reach the phloem faster than non-viruliferous insects regardless of hosts infection status (Fig. 5) where they proceed to ingest more phloem sap (Fig. 5). The phloem salivation phase was not strongly affected by the aphid and/ or plant status, but it is worth noting that viruliferous aphids (as well as non-viruliferous aphids on TuYV-infected plants) also exhibited a reduced number of probing and pathway phases, which are indicative of greater ease in accessing phloem tissues (Table S1). Since TuYV is a persistently transmitted virus that is acquired and inoculated from the phloem, more rapid phloem access and more efficient phloem feeding are likely to favor both the acquisition and inoculation processes (Mauck et al. 2012, 2018). However, these post-acquisition effects would not have been evident 
if we had only quantified vector feeding on non-infected hosts. In fact, viruliferous status drove most of the changes observed in our EPG study, largely overriding any effects of host infection status.

A similar result was observed when aphids were allowed free movement among infected and healthy hosts in retention-dispersal assays. Non-viruliferous aphids tended to remain on "release" plants, with slightly more aphids retained on TuYV-infected plants vs. non-infected plants (Fig. 4). This is consistent with prior studies documenting greater retention of M. persicae on TuYV-infected Camelina sativa (Chesnais et al. 2019a, b) as well as studies on the related species, Potato leafroll virus ([PLRV] genus Polerovirus, family Luteoviridae) and Barley yellow dwarf virus ([BYDV] genus Luteovirus, family Luteoviridae), which also report greater retention of non-viruliferous aphids on infected hosts (Jimenez-Martinez et al. 2004; Rajabaskar et al. 2014). In contrast, viruliferous aphids dispersed more readily to the "choice" plant in all assays (Fig. 4). The infection status of the release and choice plants did not play a significant role in driving viruliferous aphid distributions among plants at experiment conclusion ( $24 \mathrm{~h}$ ). Instead, viruliferous status was the significant factor. Similar experiments with BYDV, PLRV, and the related species, Cucurbit aphidborne yellows virus ([CABYV] genus Polerovirus, family Luteoviridae), have been performed to evaluate the influence of viruliferous status and host infection status on vector settling using the same full factorial design employed here (Ingwell et al. 2012; Rajabaskar et al. 2014; Carmo-Sousa et al. 2016). These experiments documented changes in vector dispersal preferences following virus acquisition over 12-h periods (Ingwell et al. 2012; Rajabaskar et al. 2014) or 48-h periods (Carmo-Sousa et al. 2016), but in all cases, vectors switched from having no preference (CABYV) or preferring to settle on virus-infected hosts (BYDV and PLRV) when non-viruliferous to having a clear settling preference for non-infected hosts when viruliferous. In our experiments, which we evaluated after $24 \mathrm{~h}$, acquisition of TuYV prior to bioassays did not reverse vector preferences but was associated with more equalized distribution of aphids across available hosts (as opposed to remaining on the release host) in the arena by $24 \mathrm{~h}$. Although the time frame differs somewhat from that used in experiments with BYDV, PLRV and CABYV, and the effects are more subdued, we can still conclude that the viruliferous aphids in our assays exhibit a greater propensity to move among adjacent plants over a biologically meaningful time period, which is expected to increase opportunities for virus transmission (Roosien et al. 2013; Shaw et al. 2017).

More extensive movement among hosts by viruliferous aphids in dispersal assays may be partially explained by the results of our locomotor activity assay, which shows that viruliferous aphids exhibit increased overall locomotor activity even in the absence of host plants (Fig. 3). This suggests that acquisition of TuYV from infected host plants may change physiological aspects underlying the capacity or motivation for foraging. We can gain some insight into the mechanisms underlying changes in viruliferous aphid activity levels by revisiting the behavioral processes of apterous aphid dispersal, which have been studied in detail for several agriculturally relevant aphid species (Hodgson 1991). When introduced to a new host environment, as in our dispersal assays, an aphid may choose to settle and remain on the host surface, or engage in locomotor activities consistent with appetitive (foraging) movement (Kennedy and Booth 1963, 1964; Fereres et al. 2007). If settling and feeding are initiated, an aphid may still choose to reinitiate locomotor activity at some later point in response to stimuli that trigger a motivation to walk (Johnson 1958; Kennedy 1965; Pettersson et al. 2007). Motivation to walk or settle is the key "switch" in these behavioral processes, and this switch can be triggered by different external stimuli, including the density of aphids in the area, host plant quality, abiotic factors (temperature, wind), presence of natural enemies, and the activities occurring just prior to host contact (e.g., flight duration) (Fereres et al. 2007; Claflin et al. 2017). Given that these factors were equalized across experiments with viruliferous and non-viruliferous aphids, we hypothesize that viruliferous apterous aphids may have a lower motivational threshold for initiating and continuing locomotor activities in response to stimuli.

Motivational threshold for dispersal via locomotor activity in response to stimuli is not well studied in aphids, but can vary with aphid life stage (Losey and Denno 1998) and diurnal rhythms (Beer et al. 2017). We know of one study besides our own that documents post-acquisition effects of a persistent circulative virus on aphid vector mobility. Levin and Irwin (1995) found that non-viruliferous alates of Rhopalosiphum padi that initiate their first flight more than $40 \mathrm{~h}$ post-eclosion can fly for longer durations than same-aged $R$. padi carrying BYDV. It should be noted that this is a different pathosystem and morph of the vector (alate vs. apterous), so it is not surprising that the results are not congruent. However, both findings are important as evidence that circulative persistent plant viruses can modify locomotor activity of aphid vectors in biologically meaningful ways. Beyond aphid-virus pathosystems, there is also evidence of vector-borne pathogens inducing similar "increased dispersal or locomotor activity" from studies on insect vectors other than aphids. Martini et al. (2015) reported that Candidatus Liberibacter asiaticus acquisition by the psyllid, Diaphorina citri, enhances flight capacity and Lima-Camara et al. (2011) found that acquisition of Dengue fever virus by Aedes aegypti increases locomotor activity. In this context, our work provides further evidence that the motivational threshold for dispersal by walking may also be reduced 
by plant virus acquisition and that this change is likely to enhance contacts between viruliferous vectors and potentially susceptible hosts.

A lower motivation threshold for movement in response to dispersal-stimulating inputs may synergize with the effects of virus acquisition on aphid fecundity, which we also observed to be positive. Viruliferous aphids produced more offspring on non-infected hosts than non-viruliferous aphids, and plant infection status did not alter this (Figs. 6 and S1). It is not possible to rule out changes in host plant infection status over the course of this experiment as playing a role in this increased fecundity; initially non-infected hosts may be inoculated and undergo physiological changes over the 4-day period of our fecundity assay. However, the biological relevance of these results remains regardless of whether the effect is partially mediated by a plant response. If we consider that viruliferous aphids more rapidly engage in feeding behaviors conducive to inoculation on non-infected hosts (Fig. 5), and their clear advantage in producing offspring (Fig. 6), there is the potential for each viruliferous adult aphid to initiate large colonies on plants that will become infected. If viruliferous aphids also have a lower motivational threshold for dispersal (Fig. 4) and a propensity to cover longer distances in shorter times (Figs. 3; S2), this may facilitate movement of now viruliferous offspring from the infected hosts to susceptible healthy hosts, which our data demonstrate are equally acceptable and supportive of fecundity as long as the aphid is viruliferous (Figs. 6; S1).

By taking a factorial approach that considered both host infection status and aphid viruliferous status and extending it beyond settling assays to a suite of metrics relevant for virus transmission, we demonstrated that post-acquisition effects of viruses on vector behavior can be important components of putative virus manipulation strategies and should figure more prominently in research moving forward. In the M. perfoliata-TuYV pathosystem, post-acquisition effects were stronger and more conducive to virus transmission than the purely plant-mediated effects observed in experiments with non-viruliferous aphids, which were limited to increases in fecundity and biomass on TuYV-infected hosts (Fig. 6) (Bogaert et al. 2020). Although we did not attempt to parse the mechanisms underlying post-acquisition effects or determine temporal durations, the thorough behavioral process work described here lays the foundation for such efforts. For example, to discern how behavioral outcomes of TuYV acquisition are mediated by direct virus effects on the aphid vs. combinations of direct and indirect (plant-mediated) effects, future work should explore movement behavior and performance of viruliferous and non-viruliferous aphids on both TuYV-susceptible hosts and non-susceptible hosts. It is also necessary to combine these experiments with approaches that separate the effects of carrying the virus from those of feeding on virus-infected plants. This could be done by purifying virions and feeding them to aphids via artificial diets within membranes (e.g., as in Ingwell et al. 2012) or through direct injection into the hemocoel (Tamborindeguy et al. 2008). Experiments employing plantfree virion delivery methods could then be combined with molecular approaches to track changes in gene expression of viruliferous and non-viruliferous aphids over the course of interactions with hosts. In addition, structural equation modeling could be employed to quantify the relative contributions of various factors in driving behavioral phenotypes conducive to virus transmission (Clark et al. 2019), and to begin exploring the importance of both pre- and post-acquisition effects in field settings (Chisholm et al. 2019). Our work presented here, and future efforts that build upon it, will ultimately provide a more complete picture of the many ways that persistently transmitted viruses alter the biology of hosts and vectors and the consequences of these effects for virus evolution and spread.

Acknowledgements This study was partly funded by The French National Agency (ANR). Project VIRAPHIPLANT ANR-14- CE19-0010-01.

Author contribution statement $\mathrm{QC}, \mathrm{MY}$ and AA conceived and designed the experiments. GCV and RC performed the experiments. QC and RC analyzed the data. QC and KM wrote the manuscript. VB and $\mathrm{AA}$ reviewed and edited the paper.

Funding This study was partly funded by The French National Agency (ANR). Project VIRAPHIPLANT ANR-14- CE19-0010-01. K. Mauck was funded in part by Hatch project funds (CA-R-ENT-5144-H).

\section{Compliance with ethical standards}

Conflict of interest The authors declare that they have no conflict of interest. The funders had no role in the design of the study, in the analyses, or interpretation of data; in the writing of the manuscript, or in the decision to publish the results.

Ethical approval All applicable institutional and/or national guidelines for the care and use of animals were followed.

\section{References}

Adams AN, Clark MF (1977) Characteristics of the microplate method of enzyme-linked immunosorbent assay for the detection of plant viruses. J Gen Virol 34:475-483. https://doi. org/10.1099/0022-1317-34-3-475

Beer K, Joschinski J, Arrazola Sastre A et al (2017) A damping circadian clock drives weak oscillations in metabolism and locomotor activity of aphids (Acyrthosiphon pisum). Sci Rep 7:1-9. https:// doi.org/10.1038/s41598-017-15014-3

Bogaert F, Marmonier A, Pichon E et al (2020) Impact of mutations in Arabidopsis thaliana metabolic pathways on polerovirus accumulation, aphid performance, and feeding behavior. Viruses 12:146. https://doi.org/10.3390/v12020146 
Carmo-Sousa M, Moreno A, Plaza M et al (2016) Cucurbit aphidborne yellows virus (CABYV) modifies the alighting, settling and probing behaviour of its vector Aphis gossypii favouring its own spread. Ann Appl Biol 169:284-297. https://doi.org/10.1111/ aab. 12300

Casteel CL, Yang C, Nanduri AC et al (2014) The NIa-Pro protein of Turnip mosaic virus improves growth and reproduction of the aphid vector, Myzus persicae (green peach aphid). Plant J 77:653663. https://doi.org/10.1111/tpj.12417

Chesnais Q, Mauck KE, Bogaert F et al (2019a) Virus effects on plant quality and vector behavior are species specific and do not depend on host physiological phenotype. J Pest Sci 92:791-804. https:// doi.org/10.1007/s10340-019-01082-z

Chesnais Q, Couty A, Uzest M et al (2019b) Plant infection by two different viruses induce contrasting changes of vectors fitness and behavior. Insect Sci 26:86-96. https://doi. org/10.1111/1744-7917.12508

Chisholm PJ, Eigenbrode SD, Clark RE et al (2019) Plant-mediated interactions between a vector and a non-vector herbivore promote the spread of a plant virus. Proc R Soc B Biol Sci 286:20191383. https://doi.org/10.1098/rspb.2019.1383

Claflin SB, Power AG, Thaler JS (2017) Aphid density and community composition differentially affect apterous aphid movement and plant virus transmission. Ecol Entomol 42:245-254. https://doi. org/10.1111/een.12381

Clark RE, Basu S, Lee BW, Crowder DW (2019) Tri-trophic interactions mediate the spread of a vector-borne plant pathogen. Ecology 53:ecy.2879. https://doi.org/10.1002/ecy.2879

Claudel P, Chesnais Q, Fouché Q et al (2018) The aphid-transmitted turnip yellows virus differentially affects volatiles emission and subsequent vector behavior in two brassicaceae plants. Int J Mol Sci 19:2316. https://doi.org/10.3390/ijms19082316

Culver JN, Padmanabhan MS (2007) Virus-induced disease: altering host physiology one interaction at a time. Annu Rev Phytopathol 45:221-243. https://doi.org/10.1146/annurev.phyto.45.06280 6.094422

Eigenbrode SD, Ding H, Shiel P, Berger PH (2002) Volatiles from potato plants infected with potato leafroll virus attract and arrest the virus vector, Myzus persicae (Homoptera: Aphididae). Proc R Soc B Biol Sci 269:455-460. https://doi.org/10.1098/ rspb.2001.1909

Eigenbrode SD, Bosque-Pérez NA, Davis TS (2018) Insect-borne plant pathogens and their vectors: ecology, evolution, and complex interactions. Annu Rev Entomol 63:169-191. https://doi. org/10.1146/annurev-ento-020117-043119

Fereres A, Moreno A (2009) Behavioural aspects influencing plant virus transmission by homopteran insects. Virus Res 141:158168. https://doi.org/10.1016/j.virusres.2008.10.020

Fereres A, Irwin ME, Kampmeier GE (2007) Aphid movement: process and consequences. Aphids as crop pests. CABI, Wallingford, pp 196-224

Giordanengo P (2014) EPG-Calc: a PHP-based script to calculate electrical penetration graph (EPG) parameters. Arthropod Plant Interact 8:163-169. https://doi.org/10.1007/s11829-014-9298-Z

Hodgson C (1991) Dispersal of apterous aphids (Homoptera: Aphididae) from their host plant and its significance. Bull Entomol Res 81:417-427. https://doi.org/10.1017/S0007485300031989

Hogenhout SA, Ammar E-D, Whitfield AE, Redinbaugh MG (2008) Insect vector interactions with persistently transmitted viruses. Annu Rev Phytopathol 46:327-359. https://doi.org/10.1146/annur ev.phyto.022508.092135

Ingwell LL, Eigenbrode SD, Bosque-Pérez NA (2012) Plant viruses alter insect behavior to enhance their spread. Sci Rep 2:1-6. https ://doi.org/10.1038/srep00578

Jimenez-Martinez ES, Bosque-Pérez NA, Berger PH et al (2004) Volatile cues influence the response of Rhopalosiphum padi
(Homoptera: Aphididae) to Barley yellow dwarf virus-infected transgenic and untransformed wheat. Environ Entomol 33:12071216. https://doi.org/10.1603/0046-225X(2004)033[1207:VCITR $\mathrm{O}] 2.0 . \mathrm{CO} ; 2$

Johnson B (1958) Factors affecting the locomotor and settling responses of alate aphids. Anim Behav 6:9-26. https://doi. org/10.1016/0003-3472(58)90004-6

Kennedy JS (1965) Co-ordination of successive activities in an aphid. Reciprocal effects of settling on flight. J Exp Biol 43:489-509

Kennedy JS, Booth CO (1963) Co-ordination of successive activities in an aphid. The effect of flight on the settling responses. J Exp Biol 40:351-369

Kennedy JS, Booth CO (1964) Co-ordination of successive activities in an aphid. Depression of settling after flight. J Exp Biol 41:805-824

Lafferty KD, Shaw JC (2013) Comparing mechanisms of host manipulation across host and parasite taxa. J Exp Biol 216:56-66. https ://doi.org/10.1242/jeb.073668

Lefèvre T, Lebarbenchon C, Gauthier-Clerc M et al (2009) The ecological significance of manipulative parasites. Trends Ecol Evol 24:41-48. https://doi.org/10.1016/j.tree.2008.08.007

Leiser RM, Ziegler-Graff V, Reutenauer A et al (1992) Agroinfection as an alternative to insects for infecting plants with beet western yellows luteovirus. Proc Natl Acad Sci 89:9136-9140. https://doi. org/10.1073/pnas.89.19.9136

Levin D, Irwin M (1995) Barley yellow dwarf luteovirus effects on tethered flight duration, wingbeat frequency, and age of maiden flight in Rhopalosiphum padi (Homoptera, Aphididae). Environ Entomol 24:306-312

Lima-Camara TN, Bruno RV, Luz PM et al (2011) Dengue infection increases the locomotor activity of Aedes aegypti females. PLoS ONE 6:e17690. https://doi.org/10.1371/journal.pone.0017690

Losey JE, Denno RF (1998) The escape response of pea aphids to foliar-foraging predators: factors affecting dropping behaviour. Ecol Entomol 23:53-61. https://doi.org/10.104 6/j.1365-2311.1998.00102.x

Lu G, Zhang T, He Y, Zhou G (2016) Virus altered rice attractiveness to planthoppers is mediated by volatiles and related to virus titre and expression of defence and volatile-biosynthesis genes. Sci Rep 6:38581. https://doi.org/10.1038/srep38581

Margaritopoulos JT, Tsourapas C, Tzortzi M et al (2005) Host selection by winged colonisers within the Myzus persicae group: a contribution towards understanding host specialisation. Ecol Entomol 30:406-418. https://doi.org/10.1111/j.0307-6946.2005.00700.x

Martini X, Hoffmann M, Coy MR et al (2015) Infection of an insect vector with a bacterial plant pathogen increases its propensity for dispersal. PLoS ONE 10:e0129373. https://doi.org/10.1371/journ al.pone. 0129373

Mauck KE, De Moraes CM, Mescher MC (2010) Deceptive chemical signals induced by a plant virus attract insect vectors to inferior hosts. Proc Natl Acad Sci 107:3600-3605. https://doi. org/10.1073/pnas.0907191107

Mauck KE, Bosque-Pérez NA, Eigenbrode SD et al (2012) Transmission mechanisms shape pathogen effects on host-vector interactions: evidence from plant viruses. Funct Ecol 26:1162-1175. https://doi.org/10.1111/j.1365-2435.2012.02026.x

Mauck KE, De Moraes CM, Mescher MC (2014) Evidence of local adaptation in plant virus effects on host-vector interactions. Integr Comp Biol 54:193-209. https://doi.org/10.1093/icb/icu012

Mauck KE, De Moraes CM, Mescher MC (2016) Effects of pathogens on sensory-mediated interactions between plants and insect vectors. Curr Opin Plant Biol 32:53-61. https://doi.org/10.1016/j. pbi.2016.06.012

Mauck KE, Chesnais Q, Shapiro LR (2018) Evolutionary determinants of host and vector manipulation by plant viruses, 1st edn. Elsevier Inc. 
Mauck KE, Kenney J, Chesnais Q (2019) Progress and challenges in identifying molecular mechanisms underlying host and vector manipulation by plant viruses. Curr Opin Insect Sci 33:7-18. https ://doi.org/10.1016/j.cois.2019.01.001

McMenemy LS, Hartley SE, MacFarlane SA et al (2012) Raspberry viruses manipulate the behaviour of their insect vectors. Entomol Exp Appl 144:56-68. https://doi.org/10.111 1/j.1570-7458.2012.01248.x

Moreno-Delafuente A, Garzo E, Moreno A, Fereres A (2013) A plant virus manipulates the behavior of its whitefly vector to enhance its transmission efficiency and spread. PLoS ONE 8:e61543. https ://doi.org/10.1371/journal.pone.0061543

Pettersson J, Tjallingii WF, Hardie J (2007) Host-plant selection and feeding. In: van Emden HF, Harrington R (eds) Aphids as crop pests. CABI, Wallingford, pp 173-195

Poulin R, Brockmann HJ, Roper TJ, et al (2010) Parasite Manipulation of Host Behavior: An Update and Frequently Asked Questions, 1 st edn. Elsevier Inc.

Powell G, Tosh CR, Hardie J (2006) Host plant selection by aphids: behavioral, evolutionary, and applied perspectives. Annu Rev Entomol 51:309-330. https://doi.org/10.1146/annur ev.ento.51.110104.151107

Prado E, Tjallingii WF (1994) Aphid activities during sieve element punctures. Entomol Exp Appl 72:157-165. https://doi. org/10.1111/j.1570-7458.1994.tb01813.x

R Core Team (2019) R: A language and environment for statistical computing. R Found. Stat. Comput. https://www.R-project.org/

Rajabaskar D, Bosque-Pérez NA, Eigenbrode SD (2014) Preference by a virus vector for infected plants is reversed after virus acquisition. Virus Res 186:32-37. https://doi.org/10.1016/j.virus res.2013.11.005
Roosien BK, Gomulkiewicz R, Ingwell LL et al (2013) Conditional vector preference aids the spread of plant pathogens: results from a model. Environ Entomol 42:1299-1308. https://doi.org/10.1603/ EN13062

Rotenberg D, Krishna Kumar NK, Ullman DE, Montero-Astúa M, Willis DK, German TL, Whitfield AE (2009) Variation in Tomato spotted wilt virus titer in Frankliniella occidentalis and its association with frequency of transmission. Phytopathology 99:404410. https://doi.org/10.1094/PHYTO-99-4-0404

Shaw AK, Peace A, Power AG, Bosque-Pérez NA (2017) Vector population growth and condition-dependent movement drive the spread of plant pathogens. Ecology 98:2145-2157. https://doi. org/10.1002/ecy.1907

Stafford CA, Walker GP, Ullman DE (2011) Infection with a plant virus modifies vector feeding behavior. Proc Natl Acad Sci U S A 108:9350-9355. https://doi.org/10.1073/pnas.1100773108

Tamborindeguy C, Gray S, Jander G (2008) Testing the physiological barriers to viral transmission in aphids using microinjection. J Vis Exp 15:e700. https://doi.org/10.3791/700

Tjallingii WF (1988) Electrical recording of stylet penetration activities. In: Aphids: Their Biology, Natural Enemies and Control. World Crop Pests., Elsevier. Amsterdam, The Netherlands, pp 95-108

Tjallingii WF, Hogen Esch T (1993) Fine structure of aphid stylet routes in plant tissues in correlation with EPG signals. Physiol Entomol 18:317-328. https://doi.org/10.1111/j.1365-3032.1993. tb00604.x

Ziegler-Graff V (2020) Molecular insights into host and vector manipulation by plant viruses. Viruses. https://doi.org/10.3390/v1203 0263 